\title{
Fixed point theory of cyclical generalized contractive conditions in partial metric spaces
}

\section{Chi-Ming Chen ${ }^{*}$}

\section{"Correspondence:}

ming@mail.nhcue.edu.tw

Department of Applied

Mathematics, National Hsinchu

University of Education, Hsinchu,

Taiwan

\begin{abstract}
The purpose of this paper is to study fixed point theorems for a mapping satisfying the cyclical generalized contractive conditions in complete partial metric spaces. Our results generalize or improve many recent fixed point theorems in the literature. MSC: $47 \mathrm{H} 10 ; 54 \mathrm{C} 60 ; 54 \mathrm{H} 25 ; 55 \mathrm{M} 20$

Keywords: fixed point; cyclic $\mathcal{C W}$-contraction; cyclic $\mathcal{M K}$-contraction; partial metric space
\end{abstract}

\section{Introduction and preliminaries}

Throughout this paper, by $\mathbb{R}^{+}$, we denote the set of all nonnegative real numbers, while $\mathbb{N}$ is the set of all natural numbers. Let $(X, d)$ be a metric space, $D$ be a subset of $X$ and $f: D \rightarrow X$ be a map. We say $f$ is contractive if there exists $\alpha \in[0,1)$ such that for all $x, y \in D$,

$$
d(f x, f y) \leq \alpha \cdot d(x, y)
$$

The well-known Banach fixed point theorem asserts that if $D=X, f$ is contractive and $(X, d)$ is complete, then $f$ has a unique fixed point in $X$. It is well known that the Banach contraction principle [1] is a very useful and classical tool in nonlinear analysis. Also, this principle has many generalizations. For instance, in 1969, Boyd and Wong [2] introduced the notion of $\Phi$-contraction. A mapping $f: X \rightarrow X$ on a metric space is called $\Phi$-contraction if there exists an upper semi-continuous function $\Phi:[0, \infty) \rightarrow[0, \infty)$ such that

$$
d(f x, f y) \leq \Phi(d(x, y)) \text { for all } x, y \in X .
$$

In 1994, Mattews [3] introduced the following notion of partial metric spaces.

Definition 1 [3] A partial metric on a nonempty set $X$ is a function $p: X \times X \rightarrow \mathbb{R}^{+}$such that for all $x, y, z \in X$,

$\left(\mathrm{p}_{1}\right) x=y$ if and only if $p(x, x)=p(x, y)=p(y, y)$;

$\left(\mathrm{p}_{2}\right) p(x, x) \leq p(x, y)$; 
$\left(\mathrm{p}_{3}\right) p(x, y)=p(y, x)$;

$\left(\mathrm{p}_{4}\right) p(x, y) \leq p(x, z)+p(z, y)-p(z, z)$.

A partial metric space is a pair $(X, p)$ such that $X$ is a nonempty set and $p$ is a partial metric on $X$.

Remark 1 It is clear that if $p(x, y)=0$, then from $\left(\mathrm{p}_{1}\right)$ and $\left(\mathrm{p}_{2}\right), x=y$. But if $x=y, p(x, y)$ may not be 0 .

Each partial metric $p$ on $X$ generates a $\mathcal{T}_{0}$ topology $\tau_{p}$ on $X$ which has as a base the family of open $p$-balls $\left\{B_{p}(x, \gamma): x \in X, \gamma>0\right\}$, where $B_{p}(x, \gamma)=\{y \in X: p(x, y)<p(x, x)+\gamma\}$ for all $x \in X$ and $\gamma>0$. If $p$ is a partial metric on $X$, then the function $d_{p}: X \times X \rightarrow \mathbb{R}^{+}$given by

$$
d_{p}(x, y)=2 p(x, y)-p(x, x)-p(y, y)
$$

is a metric on $X$.

We recall some definitions of a partial metric space as follows.

Definition 2 [3] Let $(X, p)$ be a partial metric space. Then

(1) a sequence $\left\{x_{n}\right\}$ in a partial metric space $(X, p)$ converges to $x \in X$ if and only if $p(x, x)=\lim _{n \rightarrow \infty} p\left(x, x_{n}\right)$

(2) a sequence $\left\{x_{n}\right\}$ in a partial metric space $(X, p)$ is called a Cauchy sequence if and only if $\lim _{m, n \rightarrow \infty} p\left(x_{m}, x_{n}\right)$ exists (and is finite);

(3) a partial metric space $(X, p)$ is said to be complete if every Cauchy sequence $\left\{x_{n}\right\}$ in $X$ converges, with respect to $\tau_{p}$, to a point $x \in X$ such that $p(x, x)=\lim _{m, n \rightarrow \infty} p\left(x_{m}, x_{n}\right)$;

(4) a subset $A$ of a partial metric space $(X, p)$ is closed if whenever $\left\{x_{n}\right\}$ is a sequence in $A$ such that $\left\{x_{n}\right\}$ converges to some $x \in X$, then $x \in A$.

Remark 2 The limit in a partial metric space is not unique.

Lemma $1[3,4]$

(a) $\left\{x_{n}\right\}$ is a Cauchy sequence in a partial metric space $(X, p)$ if and only if it is a Cauchy sequence in the metric space $\left(x, d_{p}\right)$;

(b) a partial metric space $(X, p)$ is complete if and only if the metric space $\left(X, d_{p}\right)$ is complete. Furthermore, $\lim _{n \rightarrow \infty} d_{p}\left(x_{n}, x\right)=0$ if and only if $p(x, x)=\lim _{n \rightarrow \infty} p\left(x_{n}, x\right)=\lim _{n \rightarrow \infty} p\left(x_{n}, x_{m}\right)$.

In 2003, Kirk, Srinivasan and Veeramani [5] introduced the following notion of the cyclic representation.

Definition 3 [5] Let $X$ be a nonempty set, $m \in \mathbb{N}$ and $f: X \rightarrow X$ be an operator. Then $X=\bigcup_{i=1}^{m} A_{i}$ is called a cyclic representation of $X$ with respect to $f$ if

(1) $A_{i}, i=1,2, \ldots, m$ are nonempty subsets of $X$;

(2) $f\left(A_{1}\right) \subset A_{2}, f\left(A_{2}\right) \subset A_{3}, \ldots, f\left(A_{m-1}\right) \subset A_{m}, f\left(A_{m}\right) \subset A_{1}$.

Kirk, Srinivasan and Veeramani [5] also proved the following theorem. 
Theorem 1 [5] Let $(X, d)$ be a complete metric space, $m \in \mathbb{N}, A_{1}, A_{2}, \ldots, A_{m}$, be closed nonempty subsets of $X$ and $X=\bigcup_{i=1}^{m} A_{i}$. Suppose that $f$ satisfies the following condition:

$$
d(f x, f y) \leq \psi(d(x, y)), \quad \text { for all } x \in A_{i}, y \in A_{i+1}, i \in\{1,2, \ldots, m\}
$$

where $\psi:[0, \infty) \rightarrow[0, \infty)$ is upper semi-continuous from the right and $0 \leq \psi(t)<t$ for $t>0$. Then $f$ has a fixed point $z \in \bigcap_{i=1}^{n} A_{i}$.

Recently, the fixed theorems for an operator $f: X \rightarrow X$ defined on a metric space $X$ with a cyclic representation of $X$ with respect to $f$ have appeared in the literature (see, e.g., [6-8]). In 2010, Păcurar and Rus [7] introduced the following notion of a cyclic weaker $\varphi$-contraction.

Definition 4 [7] Let $(X, d)$ be a metric space, $m \in \mathbb{N}, A_{1}, A_{2}, \ldots, A_{m}$ be closed nonempty subsets of $X$ and $X=\bigcup_{i=1}^{m} A_{i}$. An operator $f: X \rightarrow X$ is called a cyclic weaker $\varphi$-contraction if

(1) $X=\bigcup_{i=1}^{m} A_{i}$ is a cyclic representation of $X$ with respect to $f$;

(2) there exists a continuous, non-decreasing function $\varphi:[0, \infty) \rightarrow[0, \infty)$ with $\varphi(t)>0$ for $t \in(0, \infty)$ and $\varphi(0)=0$ such that

$$
d(f x, f y) \leq d(x, y)-\varphi(d(x, y))
$$

for any $x \in A_{i}, y \in A_{i+1}, i=1,2, \ldots, m$, where $A_{m+1}=A_{1}$.

And Păcurar and Rus [7] proved the following main theorem.

Theorem 2 [7] Let $(X, d)$ be a complete metric space, $m \in \mathbb{N}, A_{1}, A_{2}, \ldots, A_{m}$ be closed nonempty subsets of $X$ and $X=\bigcup_{i=1}^{m} A_{i}$. Suppose that $f$ is a cyclic weaker $\varphi$-contraction. Then $f$ has a fixed point $z \in \bigcap_{i=1}^{n} A_{i}$.

In the recent years, fixed point theory has developed rapidly on cyclic contraction mappings, see [9-15].

The purpose of this paper is to study fixed point theorems for a mapping satisfying the cyclical generalized contractive conditions in complete partial metric spaces. Our results generalize or improve many recent fixed point theorems in the literature.

\section{Fixed point theorems (I)}

In the section, we denote by $\Psi$ the class of functions $\psi: \mathbb{R}^{+3} \rightarrow \mathbb{R}^{+}$satisfying the following conditions:

$\left(\psi_{1}\right) \psi$ is an increasing and continuous function in each coordinate;

$\left(\psi_{2}\right)$ for $t \in \mathbb{R}^{+}, \psi(t, t, t) \leq t, \psi(t, 0,0) \leq t$ and $\psi(0,0, t) \leq t$.

Next, we denote by $\Theta$ the class of functions $\varphi: \mathbb{R}^{+} \rightarrow \mathbb{R}^{+}$satisfying the following conditions:

$\left(\varphi_{1}\right) \varphi$ is continuous and non-decreasing;

$\left(\varphi_{2}\right)$ for $t>0, \varphi(t)>0$ and $\varphi(0)=0$. 
And we denote by $\Phi$ the class of functions $\phi: \mathbb{R}^{+} \rightarrow \mathbb{R}^{+}$satisfying the following conditions:

$\left(\phi_{1}\right) \phi$ is continuous;

$\left(\phi_{2}\right)$ for $t>0, \phi(t)>0$ and $\phi(0)=0$.

We now state a new notion of cyclic $\mathcal{C W}$-contractions in partial metric spaces as follows.

Definition 5 Let $(X, p)$ be a partial metric space, $m \in \mathbb{N}, A_{1}, A_{2}, \ldots, A_{m}$ be nonempty subsets of $X$ and $Y=\bigcup_{i=1}^{m} A_{i}$. An operator $f: Y \rightarrow Y$ is called a cyclic $\mathcal{C W}$-contraction if

(1) $\bigcup_{i=1}^{m} A_{i}$ is a cyclic representation of $Y$ with respect to $f$;

(2) for any $x \in A_{i}, y \in A_{i+1}, i=1,2, \ldots, m$,

$$
\varphi(p(f x, f y)) \leq \psi(\varphi(p(x, y)), \varphi(p(x, f x)), \varphi(p(y, f y)))-\phi(M(x, y))
$$

where $\psi \in \Psi, \varphi \in \Theta, \phi \in \Phi$, and $M(x, y)=\max \{p(x, y), p(x, f x), p(y, f y)\}$.

Theorem 3 Let $(X, p)$ be a complete partial metric space, $m \in \mathbb{N}, A_{1}, A_{2}, \ldots, A_{m}$ be nonempty closed subsets of $X$ and $Y=\bigcup_{i=1}^{m} A_{i}$. Let $f: Y \rightarrow Y$ be a cyclic $\mathcal{C} \mathcal{W}$-contraction. Then $f$ has a unique fixed point $z \in \bigcap_{i=1}^{m} A_{i}$.

Proof Given $x_{0}$ and let $x_{n+1}=f x_{n}=f^{n} x_{0}$ for $n=0,1,2, \ldots$ If there exists $n_{0} \in \mathbb{N}$ such that $x_{n_{0}+1}=x_{n_{0}}$, then we finished the proof. Suppose that $x_{n+1} \neq x_{n}$ for any $n=0,1,2, \ldots$ Notice that for any $n \geq 0$, there exists $i_{n} \in\{1,2, \ldots, m\}$ such that $x_{n} \in A_{i_{n}}$ and $x_{n+1} \in A_{i_{n}+1}$.

Step 1 . We will prove that

$$
\lim _{n \rightarrow \infty} p\left(x_{n}, x_{n+1}\right)=0, \quad \text { that is, } \quad \lim _{n \rightarrow \infty} d_{p}\left(x_{n}, x_{n+1}\right)=0
$$

Using (2.1), we have

$$
\begin{aligned}
\varphi\left(p\left(x_{n}, x_{n+1}\right)\right) & =\varphi\left(p\left(f x_{n-1}, f x_{n}\right)\right) \\
& \leq \psi\left(\varphi\left(p\left(x_{n-1}, x_{n}\right)\right), \varphi\left(p\left(x_{n-1}, f x_{n-1}\right)\right), \varphi\left(p\left(x_{n}, f x_{n}\right)\right)\right)-\phi\left(M\left(x_{n-1}, x_{n}\right)\right) \\
& =\psi\left(\varphi\left(p\left(x_{n-1}, x_{n}\right)\right), \varphi\left(p\left(x_{n-1}, x_{n}\right)\right), \varphi\left(p\left(x_{n}, x_{n+1}\right)\right)\right)-\phi\left(M\left(x_{n-1}, x_{n}\right)\right),
\end{aligned}
$$

where

$$
\begin{aligned}
M\left(x_{n-1}, x_{n}\right) & =\max \left\{p\left(x_{n-1}, x_{n}\right), p\left(x_{n-1}, f x_{n-1}\right), p\left(x_{n}, f x_{n}\right)\right\} \\
& =\max \left\{p\left(x_{n-1}, x_{n}\right), p\left(x_{n-1}, x_{n}\right), p\left(x_{n}, x_{n+1}\right)\right\} .
\end{aligned}
$$

If $M\left(x_{n-1}, x_{n}\right)=p\left(x_{n}, x_{n+1}\right)$, then

$$
\begin{aligned}
\varphi\left(p\left(x_{n}, x_{n+1}\right)\right) & \leq \psi\left(\varphi\left(p\left(x_{n}, x_{n+1}\right)\right), \varphi\left(p\left(x_{n}, x_{n+1}\right)\right), \varphi\left(p\left(x_{n}, x_{n+1}\right)\right)\right)-\phi\left(p\left(x_{n}, x_{n+1}\right)\right) \\
& \leq \varphi\left(p\left(x_{n}, x_{n+1}\right)\right)-\phi\left(p\left(x_{n}, x_{n+1}\right)\right)
\end{aligned}
$$

which implies that $\phi\left(p\left(x_{n}, x_{n+1}\right)\right)=0$, and hence $p\left(x_{n}, x_{n+1}\right)=0$. This contradicts our initial assumption. 
From the above argument, we have that for each $n \in \mathbb{N}$,

$$
\varphi\left(p\left(x_{n}, x_{n+1}\right)\right) \leq \varphi\left(p\left(x_{n-1}, x_{n}\right)\right)-\phi\left(p\left(x_{n-1}, x_{n}\right)\right)
$$

and

$$
p\left(x_{n}, x_{n+1}\right)<p\left(x_{n-1}, x_{n}\right) .
$$

And since the sequence $\left\{p\left(x_{n}, x_{n+1}\right)\right\}$ is decreasing, it must converge to some $\eta \geq 0$. Taking limit as $n \rightarrow \infty$ in (2.2) and by the continuity of $\varphi$ and $\phi$, we get

$$
\varphi(\eta) \leq \varphi(\eta)-\phi(\eta)
$$

and so we conclude that $\phi(\eta)=0$ and $\eta=0$. Thus, we have

$$
\lim _{n \rightarrow \infty} p\left(x_{n}, x_{n+1}\right)=0
$$

By $\left(\mathrm{p}_{2}\right)$, we also have

$$
\lim _{n \rightarrow \infty} p\left(x_{n}, x_{n}\right)=0
$$

Since $d_{p}(x, y) \leq 2 p(x, y)-p(x, x)-p(y, y)$ for all $x, y \in X$, using (2.3) and (2.4), we obtain that

$$
\lim _{n \rightarrow \infty} d_{p}\left(x_{n}, x_{n+1}\right)=0
$$

Step 2. We show that $\left\{x_{n}\right\}$ is a Cauchy sequence in the metric space $\left(Y, d_{p}\right)$. We claim that the following result holds.

Claim For every $\varepsilon>0$, there exists $n \in \mathbb{N}$ such that if $r, q \geq n$ with $r-q=1 \bmod m$, then $d_{p}\left(x_{r}, x_{q}\right)<\varepsilon$.

Suppose the above statement is false. Then there exists $\epsilon>0$ such that for any $n \in \mathbb{N}$, there are $r_{n}, q_{n} \in \mathbb{N}$ with $r_{n}>q_{n} \geq n$ with $r_{n}-q_{n}=1 \bmod m$ satisfying

$$
d_{p}\left(x_{q_{n}}, x_{r_{n}}\right) \geq \epsilon
$$

Now, we let $n>2 m$. Then corresponding to $q_{n} \geq n$ use, we can choose $r_{n}$ in such a way it is the smallest integer with $r_{n}>q_{n} \geq n$ satisfying $r_{n}-q_{n}=1 \bmod m$ and $d_{p}\left(x_{q_{n}}, x_{r_{n}}\right) \geq \epsilon$. Therefore, $d_{p}\left(x_{q_{n}}, x_{r_{n}-m}\right) \leq \epsilon$ and

$$
\begin{aligned}
\epsilon & \leq d_{p}\left(x_{q_{n}}, x_{r_{n}}\right) \\
& \leq d_{p}\left(x_{q_{n}}, x_{r_{n}-m}\right)+\sum_{i=1}^{m} d_{p}\left(x_{r_{n-i}}, x_{r_{n-i+1}}\right) \\
& <\epsilon+\sum_{i=1}^{m} d_{p}\left(x_{r_{n-i}}, x_{r_{n-i+1}}\right) .
\end{aligned}
$$


Letting $n \rightarrow \infty$, we obtain that

$$
\lim _{n \rightarrow \infty} d_{p}\left(x_{q_{n}}, x_{r_{n}}\right)=\epsilon .
$$

On the other hand, we can conclude that

$$
\begin{aligned}
\epsilon & \leq d_{p}\left(x_{q_{n}}, x_{r_{n}}\right) \\
& \leq d_{p}\left(x_{q_{n}}, x_{q_{n+1}}\right)+d_{p}\left(x_{q_{n+1}}, x_{r_{n+1}}\right)+d_{p}\left(x_{r_{n+1}}, x_{r_{n}}\right) \\
& \leq d_{p}\left(x_{q_{n}}, x_{q_{n+1}}\right)+d_{p}\left(x_{q_{n+1}}, x_{q_{n}}\right)+d_{p}\left(x_{q_{n}}, x_{r_{n}}\right)+d_{p}\left(x_{r_{n}}, x_{r_{n+1}}\right)+d_{p}\left(x_{r_{n+1}}, x_{r_{n}}\right) .
\end{aligned}
$$

Letting $n \rightarrow \infty$, we obtain that

$$
\lim _{n \rightarrow \infty} d_{p}\left(x_{q_{n+1}}, x_{r_{n+1}}\right)=\epsilon .
$$

Since $d_{p}(x, y)=2 p(x, y)-p(x, x)-p(y, y)$ and using (2.4), (2.6) and (2.7), we have that

$$
\lim _{n \rightarrow \infty} p\left(x_{q_{n}}, x_{r_{n}}\right)=\frac{\epsilon}{2},
$$

and

$$
\lim _{n \rightarrow \infty} p\left(x_{q_{n+1}}, x_{r_{n+1}}\right)=\frac{\epsilon}{2} .
$$

Since $x_{q_{n}}$ and $x_{r_{n}}$ lie in different adjacently labeled sets $A_{i}$ and $A_{i+1}$ for certain $1 \leq i \leq m$, by using the fact that $f$ is a cyclic $\mathcal{C W}$-contraction, we have

$$
\begin{aligned}
\varphi\left(p\left(f x_{q_{n}+1}, f x_{r_{n}+1}\right)\right)= & \varphi\left(p\left(f x_{q_{n}}, f x_{r_{n}}\right)\right) \\
\leq & \psi\left(\varphi\left(p\left(x_{q_{n}}, x_{r_{n}}\right)\right), \varphi\left(p\left(x_{q_{n}}, f x_{q_{n}}\right)\right), \varphi\left(p\left(x_{r_{n}}, f x_{r_{n}}\right)\right)\right) \\
& -\phi\left(M\left(x_{q_{n}}, x_{r_{n}}\right)\right) \\
= & \psi\left(\varphi\left(p\left(x_{q_{n}}, x_{r_{n}}\right)\right), \varphi\left(p\left(x_{q_{n}}, x_{q_{n}+1}\right)\right), \varphi\left(p\left(x_{r_{n}}, x_{r_{n}+1}\right)\right)\right) \\
& -\phi\left(M\left(x_{q_{n}}, x_{r_{n}}\right)\right),
\end{aligned}
$$

where

$$
M\left(x_{q_{n}}, x_{r_{n}}\right)=\max \left\{p\left(x_{q_{n}}, x_{r_{n}}\right), p\left(x_{q_{n}}, x_{q_{n}+1}\right), p\left(x_{r_{n}}, x_{r_{n}+1}\right)\right\} .
$$

Thus, letting $n \rightarrow \infty$, we can conclude that

$$
\varphi\left(\frac{\epsilon}{2}\right) \leq \psi\left(\varphi\left(\frac{\epsilon}{2}\right), \varphi(0), \varphi(0)\right)-\phi\left(\frac{\epsilon}{2}\right) \leq \varphi\left(\frac{\epsilon}{2}\right)-\phi\left(\frac{\epsilon}{2}\right),
$$

which implies $\phi\left(\frac{\epsilon}{2}\right)=0$, that is, $\epsilon=0$. So, we get a contradiction. Therefore, our claim is proved. 
In the sequel, we will show that $\left\{x_{n}\right\}$ is a Cauchy sequence in the metric space $\left(Y, d_{p}\right)$. Let $\varepsilon>0$ be given. By our claim, there exists $n_{1} \in \mathbb{N}$ such that if $r, q \geq n_{1}$ with $r-q=1 \bmod m$, then

$$
d_{p}\left(x_{r}, x_{q}\right) \leq \frac{\varepsilon}{2}
$$

Since $\lim _{n \rightarrow \infty} d_{p}\left(x_{n}, x_{n+1}\right)=0$, there exists $n_{2} \in \mathbb{N}$ such that

$$
d_{p}\left(x_{n}, x_{n+1}\right) \leq \frac{\varepsilon}{2 m}
$$

for any $n \geq n_{2}$.

Let $r, q \geq \max \left\{n_{1}, n_{2}\right\}$ and $r>q$. Then there exists $k \in\{1,2, \ldots, m\}$ such that $r-q=$ $k \bmod m$. Therefore, $r-q+j=1 \bmod m$ for $j=m-k+1$, and so we have

$$
\begin{aligned}
d_{p}\left(x_{q}, x_{r}\right) & \leq d_{p}\left(x_{q}, x_{r+j}\right)+d_{p}\left(x_{r+j}, x_{r+j-1}\right)+\cdots+d_{p}\left(x_{r-1}, x_{r}\right) \\
& \leq \frac{\varepsilon}{2}+j \times \frac{\varepsilon}{2 m} \\
& \leq \frac{\varepsilon}{2}+m \times \frac{\varepsilon}{2 m}=\varepsilon
\end{aligned}
$$

Thus, $\left\{x_{n}\right\}$ is a Cauchy sequence in the metric space $\left(Y, d_{p}\right)$.

Step 3. We show that $f$ has a fixed point $v$ in $\bigcap_{i=1}^{m} A_{i}$.

Since $Y$ is closed, the subspace $(Y, p)$ is complete. Then from Lemma 1 , we have that $\left(Y, d_{p}\right)$ is complete. Thus, there exists $v \in X$ such that

$$
\lim _{n \rightarrow \infty} d_{p}\left(x_{n}, v\right)=0
$$

And it follows from Lemma 1 that we have

$$
p(v, v)=\lim _{n \rightarrow \infty} p\left(x_{n}, v\right)=\lim _{n, m \rightarrow \infty} p\left(x_{n}, x_{m}\right)
$$

On the other hand, since the sequence $\left\{x_{n}\right\}$ is a Cauchy sequence in the metric space $\left(Y, d_{p}\right)$, we also have

$$
\lim _{n \rightarrow \infty} d_{p}\left(x_{n}, x_{m}\right)=0
$$

Since $d_{p}(x, y)=2 p(x, y)-p(x, x)-p(y, y)$, we can deduce that

$$
\lim _{n \rightarrow \infty} p\left(x_{n}, x_{m}\right)=0
$$

Since $Y=\bigcup_{i=1}^{m} A_{i}$ is a cyclic representation of $X$ with respect to $f$, the sequence $\left\{x_{n}\right\}$ has infinite terms in each $A_{i}$ for $i \in\{1,2, \ldots, m\}$. Now, for all $i=1,2, \ldots, m$, we may take a subsequence $\left\{x_{n_{k}}\right\}$ of $\left\{x_{n}\right\}$ with $x_{n_{k}} \in A_{i-1}$ and also all converge to $v$. Using (2.10) and (2.11), we have

$$
p(v, v)=\lim _{n \rightarrow \infty} p\left(x_{n}, v\right)=\lim _{n \rightarrow \infty} p\left(x_{n_{k}}, v\right)=0 .
$$


By $(2.1)$,

$$
\begin{aligned}
\varphi\left(p\left(x_{n_{k+1}}, f v\right)\right) & =\varphi\left(p\left(f x_{n_{k}}, f v\right)\right) \\
& \leq \psi\left(\varphi\left(p\left(x_{n_{k}}, v\right)\right), \varphi\left(p\left(x_{n_{k}}, f x_{n_{k}}\right)\right), \varphi(p(v, f v))\right)-\phi\left(M\left(x_{n_{k}}, v\right)\right) \\
& =\psi\left(\varphi\left(p\left(x_{n_{k}}, v\right)\right), \varphi\left(p\left(x_{n_{k}}, x_{n_{k}+1}\right)\right), \varphi(p(v, f v))\right)-\phi\left(M\left(x_{n_{k}}, v\right)\right),
\end{aligned}
$$

where

$$
M\left(x_{n_{k}}, v\right)=\max \left\{p\left(x_{n_{k}}, v\right), p\left(x_{n_{k}}, x_{n_{k}+1}\right), p(v, f v)\right\} .
$$

Letting $k \rightarrow \infty$, we have

$$
\begin{aligned}
\varphi(p(v, f v)) & \leq \psi(\varphi(0), \varphi(0), \varphi(p(v, f v)))-\phi(p(v, f v)) \\
& \leq \varphi(p(v, f v))-\phi(p(v, f v)),
\end{aligned}
$$

which implies $\phi(p(v, f v))=0$, that is, $p(v, f v)=0$. So, $v=f v$.

Step 4. Finally, to prove the uniqueness of the fixed point, suppose that $\mu, v$ are fixed points of $f$. Then using the inequality (2.1), we obtain that

$$
\begin{aligned}
\varphi(p(\mu, \nu))= & \varphi(p(f \mu, f \nu)) \leq \psi(\varphi(p(\mu, \nu)), \varphi(p(\mu, f \mu)), \varphi(p(\nu, f v))) \\
& -\phi(M(\mu, \nu)),
\end{aligned}
$$

where

$$
M(\mu, v)=\max \{p(\mu, v), p(\mu, f \mu), p(v, f v)\}=p(\mu, v) .
$$

So, we also deduce that

$$
\begin{aligned}
\varphi(p(\mu, v)) & \leq \psi(\varphi(p(\mu, v), 0,0)) \\
& \leq \varphi(p(\mu, \nu))-\phi(p(\mu, v)),
\end{aligned}
$$

which implies that $\phi(p(\mu, v))=0$, and hence $p(\mu, v)=0$, that is, $\mu=v$. So, we complete the proof.

The following provides an example for Theorem 3.

Example 1 Let $X=[0,1]$ and $A=[0,1], B=\left[0, \frac{1}{2}\right], C=\left[0, \frac{1}{4}\right]$. We define the partial metric $p$ on $X$ by

$$
p(x, y)=\max \{x, y\} \quad \text { for all } x, y \in X,
$$

and define the function $f: X \rightarrow X$ by

$$
f(x)=\frac{x^{2}}{1+x} \text { for all } x \in X
$$


Now, we let $\varphi, \phi: \mathbb{R}^{+} \rightarrow \mathbb{R}^{+}$and $\psi: \mathbb{R}^{+3} \rightarrow \mathbb{R}^{+}$be

$$
\varphi(t)=2 t, \quad \phi(t)=\frac{2 t}{5(1+t)} \quad \text { and } \quad \psi(t)=\frac{4}{5} \cdot \max \left\{t_{1}, t_{2}, t_{3}\right\}
$$

Then $f$ is a cyclic $\mathcal{C W}$-contraction and 0 is the unique fixed point.

Proof We claim that $f$ is a cyclic $\mathcal{C W}$-contraction.

(1) Note that $f(A)=\left[0, \frac{1}{2}\right] \subset B, f(B)=\left[0, \frac{1}{6}\right] \subset C$ and $f(C)=\left[0, \frac{1}{20}\right] \subset A$. Thus, $A \cup B \cup C$ is a cyclic representation of $X$ with respect to $f$;

(2) For $x \in A$ and $y \in B$ (or, $x \in B$ and $y \in C$ ), without loss of generality, we may assume that $x \geq y$, then we have

$$
\begin{aligned}
& \varphi(p(f x, f y))=\varphi\left(p\left(\frac{x^{2}}{1+x}, \frac{y^{2}}{1+y}\right)\right)=\varphi\left(\frac{x^{2}}{1+x}\right)=\frac{2 x^{2}}{1+x} \\
& \psi(\varphi(p(x, y)), \varphi(p(x, f x)), \varphi(p(y, f y))) \\
& \quad=\psi\left(\varphi(p(x, y)), \varphi\left(p\left(x, \frac{x^{2}}{1+x}\right)\right), \varphi\left(p\left(y, \frac{y^{2}}{1+y}\right)\right)\right) \\
& \quad=\psi(\varphi(x), \varphi(x), \varphi(y)) \\
& \quad=\psi(2 x, 2 x, 2 y)=\frac{8 x}{5}
\end{aligned}
$$

and

$$
\begin{aligned}
\phi & (\max \{p(x, y), p(x, f x), p(y, f y)\}) \\
& =\phi\left(\max \left\{p(x, y), p\left(x, \frac{x^{2}}{1+x}\right), p\left(y, \frac{y^{2}}{1+y}\right)\right\}\right) \\
& =\phi(\max \{x, x, y\})=\frac{2 x}{5(1+x)} .
\end{aligned}
$$

Since

$$
\frac{2 x^{2}}{1+x} \leq \frac{8 x}{5}-\frac{2 x}{5(1+x)}
$$

we have

$$
\begin{aligned}
\varphi(p(f x, f y)) \leq & \psi(\varphi(p(x, y)), \varphi(p(x, f x)), \varphi(p(y, f y))) \\
& -\phi(\max \{p(x, y), p(x, f x), p(y, f y)\}) .
\end{aligned}
$$

On the other hand, for $x \in C$ and $y \in A$, without loss of generality, we may assume that $x \leq y$, then it is easy to get the above inequality.

Note that Example 1 satisfies all of the hypotheses of Theorem 3, and we get that 0 is the unique fixed point.

\section{Fixed point theorems (II)}

In this article, we also recall the notion of a Meir-Keeler function (see [16]). A function $\phi:[0, \infty) \rightarrow[0, \infty)$ is said to be a Meir-Keeler function if for each $\eta>0$, there exists $\delta>0$ 
such that for $t \in[0, \infty)$ with $\eta \leq t<\eta+\delta$, we have $\phi(t)<\eta$. We now introduce a new notion of a weaker Meir-Keeler function $\phi:[0, \infty) \rightarrow[0, \infty)$ in a partial metric space $(X, p)$ as follows.

Definition 6 Let $(X, p)$ be a partial metric space. We call $\phi:[0, \infty) \rightarrow[0, \infty)$ a weaker Meir-Keeler function in $X$ if for each $\eta>0$, there exists $\delta>0$ such that for $x, y \in X$ with $\eta \leq p(x, y)<\eta+\delta$, there exists $n_{0} \in \mathbb{N}$ such that $\phi^{n_{0}}(p(x, y))<\eta$.

In the section, we denote by $\Phi$ the class of weaker Meir-Keeler functions $\phi: \mathbb{R}^{+} \rightarrow \mathbb{R}^{+}$ in a partial metric space in $(X, p)$ satisfying the following conditions:

$\left(\phi_{1}\right) \phi(t)>0$ for $t>0, \phi(0)=0$;

$\left(\phi_{2}\right)\left\{\phi^{n}(t)\right\}_{n \in \mathbb{N}}$ is decreasing;

$\left(\phi_{3}\right)$ for $t_{n} \in[0, \infty)$,

(a) if $\lim _{n \rightarrow \infty} t_{n}=\gamma>0$, then $\lim _{n \rightarrow \infty} \phi\left(t_{n}\right)<\gamma$ and

(b) if $\lim _{n \rightarrow \infty} t_{n}=0$, then $\lim _{n \rightarrow \infty} \phi\left(t_{n}\right)=0$.

And we denote by the class $\Psi$ of functions $\psi: \mathbb{R}^{+} \rightarrow \mathbb{R}^{+}$a continuous function satisfying $\psi(t)>0$ for $t>0, \psi(0)=0$.

First, we state a new notion of cyclic $\mathcal{M K}$-contractions in partial metric spaces as follows.

Definition 7 Let $(X, p)$ be a partial metric space, $m \in \mathbb{N}, A_{1}, A_{2}, \ldots, A_{m}$ be nonempty subsets of $X$ and $Y=\bigcup_{i=1}^{m} A_{i}$. An operator $f: Y \rightarrow Y$ is called a cyclic $\mathcal{M K}$-contraction if

(1) $\bigcup_{i=1}^{m} A_{i}$ is a cyclic representation of $Y$ with respect to $f$

(2)

for any $x \in A_{i}, y \in A_{i+1}, i=1,2, \ldots, m$,

$$
p(f x, f y) \leq \phi(p(x, y))-\psi(p(x, y))
$$

where

$A_{m+1}=A_{1}, \phi \in \Phi$ and $\psi \in \Psi$.

Theorem 4 Let $(X, p)$ be a complete partial metric space, $m \in \mathbb{N}, A_{1}, A_{2}, \ldots, A_{m}$ be nonempty closed subsets of $X$ and $Y=\bigcup_{i=1}^{m} A_{i}$. Let $f: Y \rightarrow Y$ be a cyclic $\mathcal{M K}$-contraction. Then $f$ has a unique fixed point $z \in \bigcap_{i=1}^{m} A_{i}$.

Proof Given $x_{0}$ and let $x_{n+1}=f x_{n}=f^{n} x_{0}$, for $n=0,1,2, \ldots$ If there exists $n_{0} \in \mathbb{N}$ such that $x_{n_{0}+1}=x_{n_{0}}$, then we finished the proof. Suppose that $x_{n+1} \neq x_{n}$ for any $n=0,1,2, \ldots$. Notice that for any $n \geq 0$, there exists $i_{n} \in\{1,2, \ldots, m\}$ such that $x_{n} \in A_{i_{n}}$ and $x_{n+1} \in A_{i_{n}+1}$. Then by (3.1), we have

$$
p\left(x_{n}, x_{n+1}\right)=p\left(f x_{n-1}, f x_{n}\right) \leq \phi\left(p\left(x_{n-1}, x_{n}\right)\right)-\psi\left(p\left(x_{n-1}, x_{n}\right)\right) .
$$

Step 1. We will prove that

$$
\lim _{n \rightarrow \infty} p\left(x_{n}, x_{n+1}\right)=0, \quad \text { that is, } \quad \lim _{n \rightarrow \infty} d_{p}\left(x_{n}, x_{n+1}\right)=0 .
$$


Since $f$ is a cyclic $\mathcal{M K}$-contraction, we can conclude that

$$
\begin{aligned}
p\left(x_{n}, x_{n+1}\right) & \leq \phi\left(p\left(x_{n-1}, x_{n}\right)\right) \\
& \leq \phi\left(\phi\left(p\left(x_{n-2}, x_{n-1}\right)\right)=\phi^{2}\left(p\left(x_{n-2}, x_{n-1}\right)\right)\right. \\
& \leq \cdots \\
& \leq \phi^{n}\left(p\left(x_{0}, x_{1}\right)\right) .
\end{aligned}
$$

Since $\left\{\phi^{n}\left(p\left(x_{0}, x_{1}\right)\right)\right\}_{n \in \mathbb{N}}$ is decreasing, it must converge to some $\eta \geq 0$. We claim that $\eta=0$. On the contrary, assume that $0<\eta$. Then by the definition of a weaker Meir-Keeler function $\phi$, there exists $\delta>0$ such that for $x_{0}, x_{1} \in X$ with $\eta \leq p\left(x_{0}, x_{1}\right)<\delta+\eta$, there exists $n_{0} \in \mathbb{N}$ such that $\phi^{n_{0}}\left(p\left(x_{0}, x_{1}\right)\right)<\eta$. Since $\lim _{n \rightarrow \infty} \phi^{n}\left(p\left(x_{0}, x_{1}\right)\right)=\eta$, there exists $k_{0} \in \mathbb{N}$ such that $\eta \leq \phi^{k}\left(p\left(x_{0}, x_{1}\right)\right)<\delta+\eta$, for all $k \geq k_{0}$. Thus, we conclude that $\phi^{k_{0}+n_{0}}\left(p\left(x_{0}, x_{1}\right)\right)<\eta$. So, we get a contradiction. Therefore, $\lim _{n \rightarrow \infty} \phi^{n}\left(p\left(x_{0}, x_{1}\right)\right)=0$, and so we have

$$
\lim _{n \rightarrow \infty} p\left(x_{n}, x_{n+1}\right)=0
$$

By $\left(\mathrm{p}_{2}\right)$, we also have

$$
\lim _{n \rightarrow \infty} p\left(x_{n}, x_{n}\right)=0
$$

Since $d_{p}(x, y) \leq 2 p(x, y)-p(x, x)-p(y, y)$ for all $x, y \in X$, using (3.2) and (3.3), we obtain that

$$
\lim _{n \rightarrow \infty} d_{p}\left(x_{n}, x_{n+1}\right)=0
$$

Step 2. We show that $\left\{x_{n}\right\}$ is a Cauchy sequence in the metric space $\left(Y, d_{p}\right)$. We claim that the following result holds.

Claim For every $\varepsilon>0$, there exists $n \in \mathbb{N}$ such that if $r, q \geq n$ with $r-q=1 \bmod m$, then $d_{p}\left(x_{r}, x_{q}\right)<\varepsilon$.

Suppose the above statement is false. Then there exists $\epsilon>0$ such that for any $n \in \mathbb{N}$, there are $r_{n}, q_{n} \in \mathbb{N}$ with $r_{n}>q_{n} \geq n$ with $r_{n}-q_{n}=1 \bmod m$ satisfying

$$
d_{p}\left(x_{q_{n}}, x_{r_{n}}\right) \geq \epsilon .
$$

Now, we let $n>2 m$. Then corresponding to $q_{n} \geq n$ use, we can choose $r_{n}$ in such a way it is the smallest integer with $r_{n}>q_{n} \geq n$ satisfying $r_{n}-q_{n}=1 \bmod m$ and $d_{p}\left(x_{q_{n}}, x_{r_{n}}\right) \geq \epsilon$. Therefore, $d_{p}\left(x_{q_{n}}, x_{r_{n}-m}\right) \leq \epsilon$ and

$$
\begin{aligned}
\epsilon & \leq d_{p}\left(x_{q_{n}}, x_{r_{n}}\right) \\
& \leq d_{p}\left(x_{q_{n}}, x_{r_{n}-m}\right)+\sum_{i=1}^{m} d_{p}\left(x_{r_{n-i}}, x_{r_{n-i+1}}\right) \\
& <\epsilon+\sum_{i=1}^{m} d_{p}\left(x_{r_{n-i}}, x_{r_{n-i+1}}\right) .
\end{aligned}
$$


Letting $n \rightarrow \infty$, we obtain that

$$
\lim _{n \rightarrow \infty} d_{p}\left(x_{q_{n}}, x_{r_{n}}\right)=\epsilon
$$

On the other hand, we can conclude that

$$
\begin{aligned}
\epsilon & \leq d_{p}\left(x_{q_{n}}, x_{r_{n}}\right) \\
& \leq d_{p}\left(x_{q_{n}}, x_{q_{n+1}}\right)+d_{p}\left(x_{q_{n+1}}, x_{r_{n+1}}\right)+d_{p}\left(x_{r_{n+1}}, x_{r_{n}}\right) \\
& \leq d_{p}\left(x_{q_{n}}, x_{q_{n+1}}\right)+d_{p}\left(x_{q_{n+1}}, x_{q_{n}}\right)+d_{p}\left(x_{q_{n}}, x_{r_{n}}\right)+d_{p}\left(x_{r_{n}}, x_{r_{n+1}}\right)+d_{p}\left(x_{r_{n+1}}, x_{r_{n}}\right) .
\end{aligned}
$$

Letting $n \rightarrow \infty$, we obtain that

$$
\lim _{n \rightarrow \infty} d_{p}\left(x_{q_{n+1}}, x_{r_{n+1}}\right)=\epsilon .
$$

Since $d_{p}(x, y)=2 p(x, y)-p(x, x)-p(y, y)$ and using (3.5) and (3.6), we have that

$$
\lim _{n \rightarrow \infty} p\left(x_{q_{n}}, x_{r_{n}}\right)=\frac{\epsilon}{2},
$$

and

$$
\lim _{n \rightarrow \infty} p\left(x_{q_{n+1}}, x_{r_{n+1}}\right)=\frac{\epsilon}{2} .
$$

Since $x_{q_{n}}$ and $x_{r_{n}}$ lie in different adjacently labeled sets $A_{i}$ and $A_{i+1}$ for certain $1 \leq i \leq m$, by using the fact that $f$ is a cyclic $\mathcal{M K}$-contraction, we have

$$
p\left(x_{q_{n+1}}, x_{r_{n+1}}\right)=p\left(f x_{q_{n}}, f x_{r_{n}}\right) \leq \phi\left(p\left(x_{q_{n}}, x_{r_{n}}\right)\right)-\psi\left(p\left(x_{q_{n}}, x_{r_{n}}\right)\right) .
$$

Letting $n \rightarrow \infty$, by using the condition $\phi_{3}$ of the function $\phi$, we obtain that

$$
\frac{\epsilon}{2} \leq \frac{\epsilon}{2}-\psi\left(\frac{\epsilon}{2}\right)
$$

and consequently, $\psi\left(\frac{\epsilon}{2}\right)=0$. By the definition of a function $\psi$, we get $\epsilon=0$ which is a contraction. Therefore, our claim is proved.

In the sequel, we will show that $\left\{x_{n}\right\}$ is a Cauchy sequence in the metric space $\left(Y, d_{p}\right)$. Let $\varepsilon>0$ be given. By our claim, there exists $n_{1} \in \mathbb{N}$ such that if $r, q \geq n_{1}$ with $r-q=1 \bmod m$, then

$$
d_{p}\left(x_{r}, x_{q}\right) \leq \frac{\varepsilon}{2}
$$

Since $\lim _{n \rightarrow \infty} d_{p}\left(x_{n}, x_{n+1}\right)=0$, there exists $n_{2} \in \mathbb{N}$ such that

$$
d_{p}\left(x_{n}, x_{n+1}\right) \leq \frac{\varepsilon}{2 m}
$$

for any $n \geq n_{2}$. 
Let $r, q \geq \max \left\{n_{1}, n_{2}\right\}$ and $r>q$. Then there exists $k \in\{1,2, \ldots, m\}$ such that $r-q=$ $k \bmod m$. Therefore, $r-q+j=1 \bmod m$ for $j=m-k+1$, and so we have

$$
\begin{aligned}
d_{p}\left(x_{q}, x_{r}\right) & \leq d_{p}\left(x_{q}, x_{r+j}\right)+d_{p}\left(x_{r+j}, x_{r+j-1}\right)+\cdots+d_{p}\left(x_{r-1}, x_{r}\right) \\
& \leq \frac{\varepsilon}{2}+j \times \frac{\varepsilon}{2 m} \\
& \leq \frac{\varepsilon}{2}+m \times \frac{\varepsilon}{2 m}=\varepsilon .
\end{aligned}
$$

Thus, $\left\{x_{n}\right\}$ is a Cauchy sequence in the metric space $\left(Y, d_{p}\right)$.

Step 3. We show that $f$ has a fixed point $v$ in $\bigcap_{i=1}^{m} A_{i}$.

Since $Y$ is closed, the subspace $(Y, p)$ is complete. Then from Lemma 1 , we have that $\left(Y, d_{p}\right)$ is complete. Thus, there exists $v \in X$ such that

$$
\lim _{n \rightarrow \infty} d_{p}\left(x_{n}, v\right)=0
$$

And it follows from Lemma 1 that we have

$$
p(v, v)=\lim _{n \rightarrow \infty} p\left(x_{n}, v\right)=\lim _{n, m \rightarrow \infty} p\left(x_{n}, x_{m}\right)
$$

On the other hand, since the sequence $\left\{x_{n}\right\}$ is a Cauchy sequence in the metric space $\left(Y, d_{p}\right)$, we also have

$$
\lim _{n \rightarrow \infty} d_{p}\left(x_{n}, x_{m}\right)=0
$$

Since $d_{p}(x, y)=2 p(x, y)-p(x, x)-p(y, y)$, we can deduce that

$$
\lim _{n \rightarrow \infty} p\left(x_{n}, x_{m}\right)=0
$$

Since $Y=\bigcup_{i=1}^{m} A_{i}$ is a cyclic representation of $X$ with respect to $f$, the sequence $\left\{x_{n}\right\}$ has infinite terms in each $A_{i}$ for $i \in\{1,2, \ldots, m\}$. Now, for all $i=1,2, \ldots, m$, we may take a subsequence $\left\{x_{n_{k}}\right\}$ of $\left\{x_{n}\right\}$ with $x_{n_{k}} \in A_{i-1}$ and also all converge to $v$. Using (3.9) and (3.10), we have

$$
p(v, v)=\lim _{n \rightarrow \infty} p\left(x_{n}, v\right)=\lim _{n \rightarrow \infty} p\left(x_{n_{k}}, v\right)=0 .
$$

By (3.1),

$$
\begin{aligned}
p\left(x_{n_{k+1}}, f v\right) & =p\left(f x_{n_{k}}, f v\right) \\
& \leq \phi\left(p\left(x_{n_{k}}, v\right)\right)-\psi\left(p\left(x_{n_{k}}, v\right)\right) \\
& \leq \phi\left(p\left(x_{n_{k}}, v\right)\right) .
\end{aligned}
$$

Letting $k \rightarrow \infty$, we have

$$
p(\nu, f v) \leq 0,
$$

and so $v=f v$. 
Step 4. Finally, to prove the uniqueness of the fixed point, let $\mu$ be another fixed point of $f$ in $\bigcap_{i=1}^{m} A_{i}$. By the cyclic character of $f$, we have $\mu, v \in \bigcap_{i=1}^{n} A_{i}$. Since $f$ is a cyclic weaker $\mathcal{M K}$-contraction, we have

$$
\begin{aligned}
p(\nu, \mu) & =p(\nu, f \mu) \\
& =\lim _{n \rightarrow \infty} p\left(x_{n_{k+1}}, f \mu\right) \\
& =\lim _{n \rightarrow \infty} p\left(f x_{n_{k}}, f \mu\right) \\
& \leq \lim _{n \rightarrow \infty}\left[\phi\left(p\left(x_{n_{k}}, \mu\right)\right)-\psi\left(p\left(x_{n_{k}}, \mu\right)\right)\right] \\
& \leq p(v, \mu)-\psi(p(v, \mu)),
\end{aligned}
$$

and we can conclude that

$$
\psi(p(\nu, \mu))=0,
$$

which implies $p(v, \mu)=0$. So, we have $\mu=v$. We complete the proof.

The following provides an example for Theorem 4.

Example 2 Let $X=[0,1]$ and $A=[0,1], B=\left[0, \frac{1}{2}\right], C=\left[0, \frac{1}{4}\right]$. We define the partial metric $p$ on $X$ by

$$
p(x, y)=\max \{x, y\} \quad \text { for all } x, y \in X,
$$

and define the function $f: X \rightarrow X$ by

$$
f(x)=\frac{x^{2}}{1+x} \quad \text { for all } x \in X
$$

Now, we let $\psi, \phi: \mathbb{R}^{+} \rightarrow \mathbb{R}^{+}$be

$$
\phi(t)=\frac{4 t}{5} \quad \text { and } \quad \psi(t)=\frac{t}{5(1+t)} .
$$

Then $f$ is a cyclic $\mathcal{M K}$-contraction and 0 is the unique fixed point.

By Theorem 4, it is easy to get the following corollary.

Corollary 1 Let $(X, p)$ be a complete partial metric space, $m \in \mathbb{N}, A_{1}, A_{2}, \ldots, A_{m}$ be nonempty closed subsets of $X, Y=\bigcup_{i=1}^{m} A_{i}$ and let $f: Y \rightarrow Y$. Assume that

(1) $\bigcup_{i=1}^{m} A_{i}$ is a cyclic representation of $Y$ with respect to $f$;

(2) for any $x \in A_{i}, y \in A_{i+1}, i=1,2, \ldots, m$,

$$
p(f x, f y) \leq \phi(p(x, y)),
$$

where $A_{m+1}=A_{1}$ and $\phi \in \Phi$.

Then $f$ has a unique fixed point $z \in \bigcap_{i=1}^{m} A_{i}$. 


\section{Competing interests}

The author declares that they have no competing interests.

\section{Acknowledgements}

The authors would like to thank referee(s) for many useful comments and suggestions for the improvement of the paper.

Received: 6 November 2012 Accepted: 13 January 2013 Published: 28 January 2013

\section{References}

1. Banach, S: Sur les operations dans les ensembles abstraits et leur application aux equations integerales. Fundam. Math. 3, 133-181 (1922)

2. Boyd, DW, Wong, SW: On nonlinear contractions. Proc. Am. Math. Soc. 20, 458-464 (1969)

3. Mattews, SG: Partial metric topology. In: Proc. 8th Summer of Conference on General Topology and Applications. Ann. New York Acad. Sci., vol. 728, pp. 183-197 (1994)

4. Oltra, S, Valero, O: Banach's fixed point theorem for partial metric spaces. Rend. Ist. Mat. Univ. Trieste 36, 17-26 (2004)

5. Kirk, WA, Srinivasan, PS, Veeramani, P: Fixed points for mappings satisfying cyclical contractive conditions. Fixed Point Theory Appl. 4(1), 79-89 (2003)

6. Karapinar, E: Fixed point theory for cyclic weaker $\phi$-contraction. Appl. Math. Lett. 24(6), 822-825 (2011)

7. Păcurar, M, Rus, IA: Fixed point theory for cyclic $\varphi$-contractions. Nonlinear Anal. 72(3-4), 2683-2693 (2010)

8. Rus, IA: Cyclic representations and fixed points. Ann. "Tiberiu Popoviciu” Sem. Funct. Equ. Approx. Convexity 3, $171-178(2005)$

9. Agarwal, RP, Alghamdi, MA, Shahzad, N: Fixed point theory for cyclic generalized contractions in partial metric spaces Fixed Point Theory Appl. 2012, Article ID 40 (2012)

10. Di Bari, C, Suzuki, T, Vetro, C: Best proximity for cyclic Meir-Keeler contractions. Nonlinear Anal. 69, 3790-3794 (2008)

11. Karpagam, S, Agrawal, S: Best proximity point theorems for $p$-cyclic Meir-Keeler contractions. Fixed Point Theory Appl. 2009, Article ID 197308 (2009)

12. Karpagam, S, Agrawal, S: Best proximity point theorems for cyclic orbital Meir-Keeler contraction maps. Nonlinear Anal. 74, 1040-1046 (2010)

13. Karapinar, E, Erhan, IM: Best proximity point on different type contraction. Inf. Sci. Appl. Math. 5, $342-353$ (2011)

14. Karapinar, E, Erhan, IM: Fixed point theorem for cyclic maps on partial metric spaces. Inf. Sci. Appl. Math. 6, 239-244 (2012)

15. Karapinar, E, Erhan, IM: Cyclic contractions and fixed point theorems. Filomat 26, 777-782 (2012)

16. Meir, A, Keeler, E: A theorem on contraction mappings. J. Math. Anal. Appl. 28, 326-329 (1969)

doi:10.1186/1687-1812-2013-17

Cite this article as: Chen: Fixed point theory of cyclical generalized contractive conditions in partial metric spaces.

Fixed Point Theory and Applications 2013 2013:17.

\section{Submit your manuscript to a SpringerOpen ${ }^{\circ}$ journal and benefit from:}

- Convenient online submission

- Rigorous peer review

Immediate publication on acceptance

- Open access: articles freely available online

- High visibility within the field

- Retaining the copyright to your article 